\title{
The protective effect of Schiff and Mannich bases on steel corrosion in neutral media
}

\author{
A.I. Altsybeeva, ${ }^{1}$ M.A. Pletnev, ${ }^{2}{ }^{2}$ S.M. Reshetnikov ${ }^{3}$ and I.B. Shirobokov ${ }^{\dagger 3}$

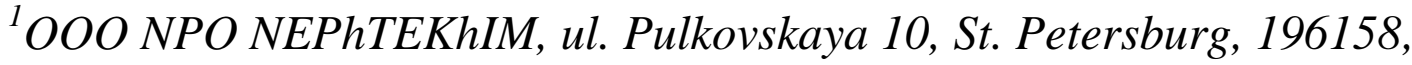 \\ Russian Federation \\ ${ }^{2}$ Kalashnikov Izhevsk State Technical University, 7, Studencheskaya St., Izhevsk, 426069, \\ Russian Federation \\ ${ }^{3}$ Udmurt State University, 1, Universitetskaya St., Izhevsk, 426034, Russian Federation \\ *E-mail:pletnevm@list.ru
}

\begin{abstract}
The protective effect of Schiff and Mannich bases - morpholine and benzotriazole derivatives - on steel corrosion in neutral media has been studied. These substances were previously investigated as inhibitors of atmospheric corrosion. Protective properties were calculated by the effect of the inhibitors on the anodic dissolution rate. The electrochemical measurement was carried out in a mixed electrolyte of $70 \mathrm{mg} / \mathrm{dm}^{3} \mathrm{NaCl}+80 \mathrm{mg} / \mathrm{dm}^{3}$ $\mathrm{Na}_{2} \mathrm{SO}_{4}$, containing $10 \%$ isopropyl alcohol to ensure the solubility of the inhibitors. It has been found that all studied inhibitors - derivatives of morpholine and benzotriazole are more effective than unsubstituted starting compounds. The paper analyzes the dependencies of corrosion inhibitors of this class on a complex of physicochemical properties, such as saturated vapor pressure, electron density on heteroatoms, ionization potentials (PI) of inhibitors, and Hansch hydrophobic constants. Based on literature and experimental data, the protective effect of the morpholine and benzotriazole derivatives investigated in the present work is due to the hydrophobicity effect of the molecules. The decrease in the rate of anodic dissolution in the presence of inhibitors is due to a decrease in the dielectric constant of the electrode layer during the adsorption of inhibitors.
\end{abstract}

Key words: Schiff and Mannich bases, inhibition, steel corrosion, adsorption, hydrophobicity effect.

Received: October 5, 2018. Published: January 25, 2019

doi: $\underline{10.17675 / 2305-6894-2019-8-1-6}$

\section{Introduction}

Schiff and Mannich bases are known to be volatile inhibitors of atmospheric corrosion of metals [1]. However, compounds of this class can exhibit protective properties in neutral aqueous solutions [2]. Determination of the protective properties of volatile inhibitors in neutral aqueous solutions is used for a preliminary assessment of their effectiveness $[2,3]$. In this work, we studied the protective effect of Schiff and Mannich bases, derivatives of 
morpholine and benzotriazole, previously studied as inhibitors of atmospheric corrosion [3], during corrosion of low carbon steel in neutral aqueous solutions.

\section{Experimental}

Commercial grade St.20 low carbon was used in the study. The working electrode was polished with emery papers, washed with water and degreased with acetone.

The electrochemical measurement was carried out in a mixed electrolyte of $70 \mathrm{mg} / \mathrm{dm}^{3}$ $\mathrm{NaCl}+80 \mathrm{mg} / \mathrm{dm}^{3} \mathrm{Na}_{2} \mathrm{SO}_{4}$, containing $10 \%$ isopropyl alcohol to ensure the solubility of the inhibitors. All solutions were prepared from analytical reagent grade chemicals using distilled water. Polarization was performed using ICP-Pro potentiostat at a potential scan rate of $1 \mathrm{mV} / \mathrm{s}$. A saturated $\mathrm{Ag} / \mathrm{AgCl}$ electrode (SSE) was used as reference electrode. The measured electrode potential was converted from its SSE-based scale to the standard scale (HNE).

In the work, the inhibitors studied included morpholine and its derivatives: VNH-L20 (dimorpholine phenylmethane); VNH-L-21 (1-(phenyl)-1-(morpholinomethyl)cyclohexylamine); VNH-L-112 (dimorpholinomethane); VNH-L-113 (1morpholinomethylcyclohexylamine), as well as benzotriazole and its derivatives: VNH-L405 (1-cyclohexylaminomethylbenzotriazole); VNH-L-406 (1-morpholinomethylbenzotriazole); VNH-L-407 (1-(phenyl)-1-(cyclohexylaminomethyl)benzotriazole); BHH-L-408 (1-(phenyl)-1-(morpholinomethyl)benzotriazole). The concentration of the inhibitors studied was varied in the range from 0.5 to $100 \mathrm{mg} / \mathrm{dm}^{3}$.

\section{Results and discussion}

It was previously shown that compounds of this class in neutral low-mineralized media are inhibitors of the anodic dissolution [4], therefore, in this work, to determine the protective effect, the effect of inhibitors on the anodic dissolution rate of steel was calculated at $-0.1 \mathrm{~V}$.

Inhibitor efficiency is determined in accordance to GOST 9.506-87:

$$
Z=\frac{i_{0}-i}{i_{0}} \cdot 100,
$$

or inhibition coefficient:

$$
\gamma=\frac{i_{0}}{i},
$$

where $i$ and $i_{0}$ - anodic current density with/without inhibitors.

Figure 1 shows that with an increase in the concentration of the inhibitor VNH-L-112, the corrosion potential shifts to the area of the anodic potentials, which indicates that the anodic dissolution reaction is mainly inhibited. Similarly shaped polarization curves were obtained for all inhibitors studied, including benzotriazole and morpholine. 


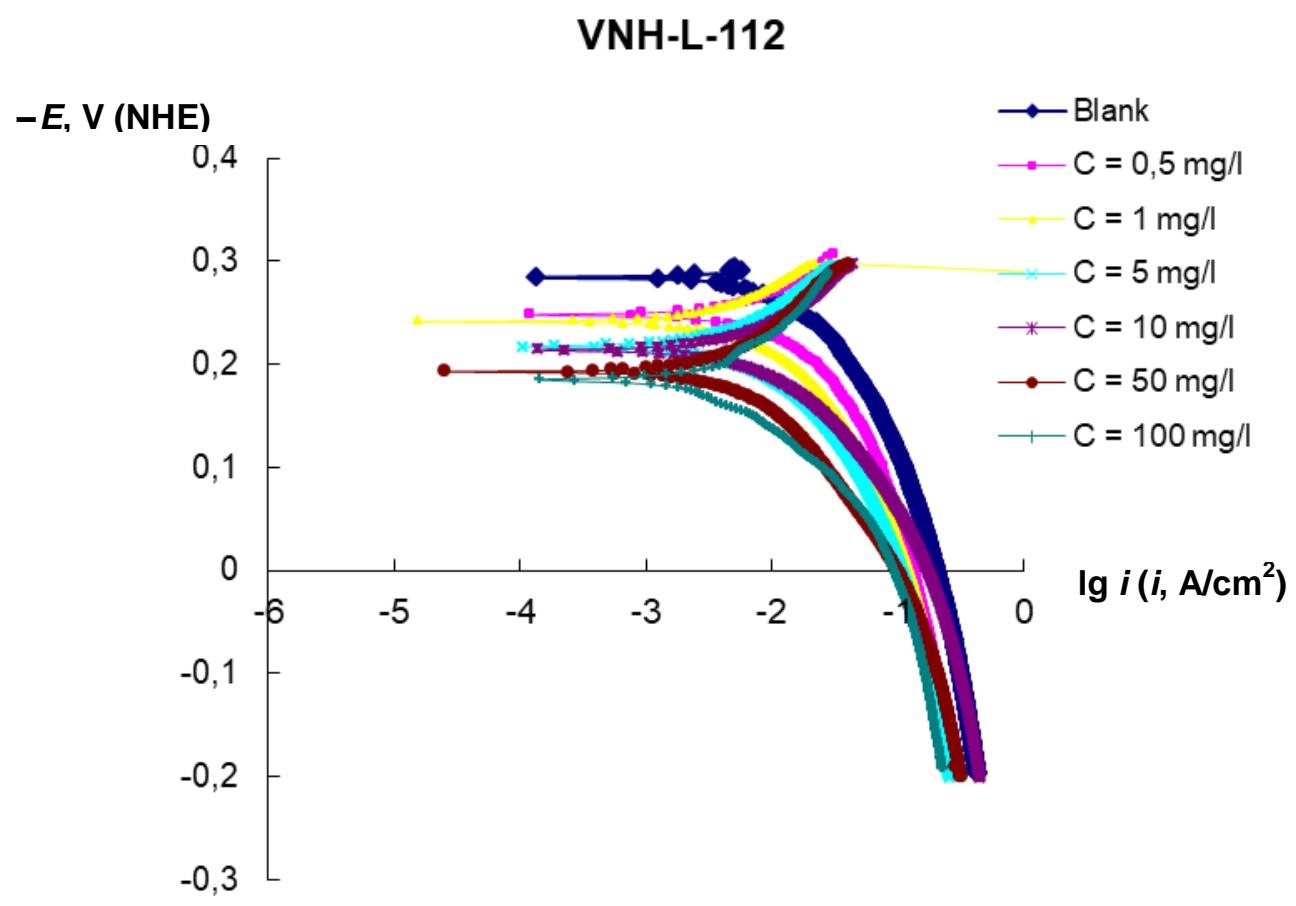

Figure 1. Polarization curves of St.20 in $70 \mathrm{mg} / \mathrm{l} \mathrm{NaCl}+80 \mathrm{mg} / \mathrm{Na}_{2} \mathrm{SO}_{4}$ plus $10 \%$ isopropyl alcohol, with the addition of an inhibitor of VNH-L-112 of various concentrations.

All inhibitors studied - morpholine and benzotriazole derivatives - are more effective than the substituted starting compounds (Figures 2,3). In work [3], the analysis of dependencies of inhibitors of this type as volatile corrosion inhibitors on a set of physicochemical properties, such as saturated vapor pressure, electron density on heteroatoms, ionization potentials (PI) of the inhibitors, Hansh's hydrophobic constants, did not give an unambiguous choice in favor of either of the parameters. In the present work, the effect of the same physicochemical properties as in [3] on the protective effect of the studied inhibitors in neutral media was analyzed.

From Figures 4 and 5, it follows that the protective effect of inhibitors of this series is mainly determined by the hydrophobicity of the substituents (the correlation coefficient is $0.887)$. The highest protective ability $(Z=89.0 \%)$ of morpholine derivatives is shown by the inhibitor VNH-L 113, which has the highest hydrophobicity constant $(\pi=2.33)$. In the series of benzotriazole derivatives, a similar dependence of the logarithm of the inhibition coefficient on the Hansch constant with a correlation coefficient of 0.981 is observed (Figure 6). There is little or no correlation between the other physicochemical parameters of inhibitors of this series and the protective effect. VNH-L-407 has the highest hydrophobicity constant among the studied benzotriazole derivatives $(\pi=3.44)$, which, apparently, determines its high protective ability $(Z=95.5 \%)$. 


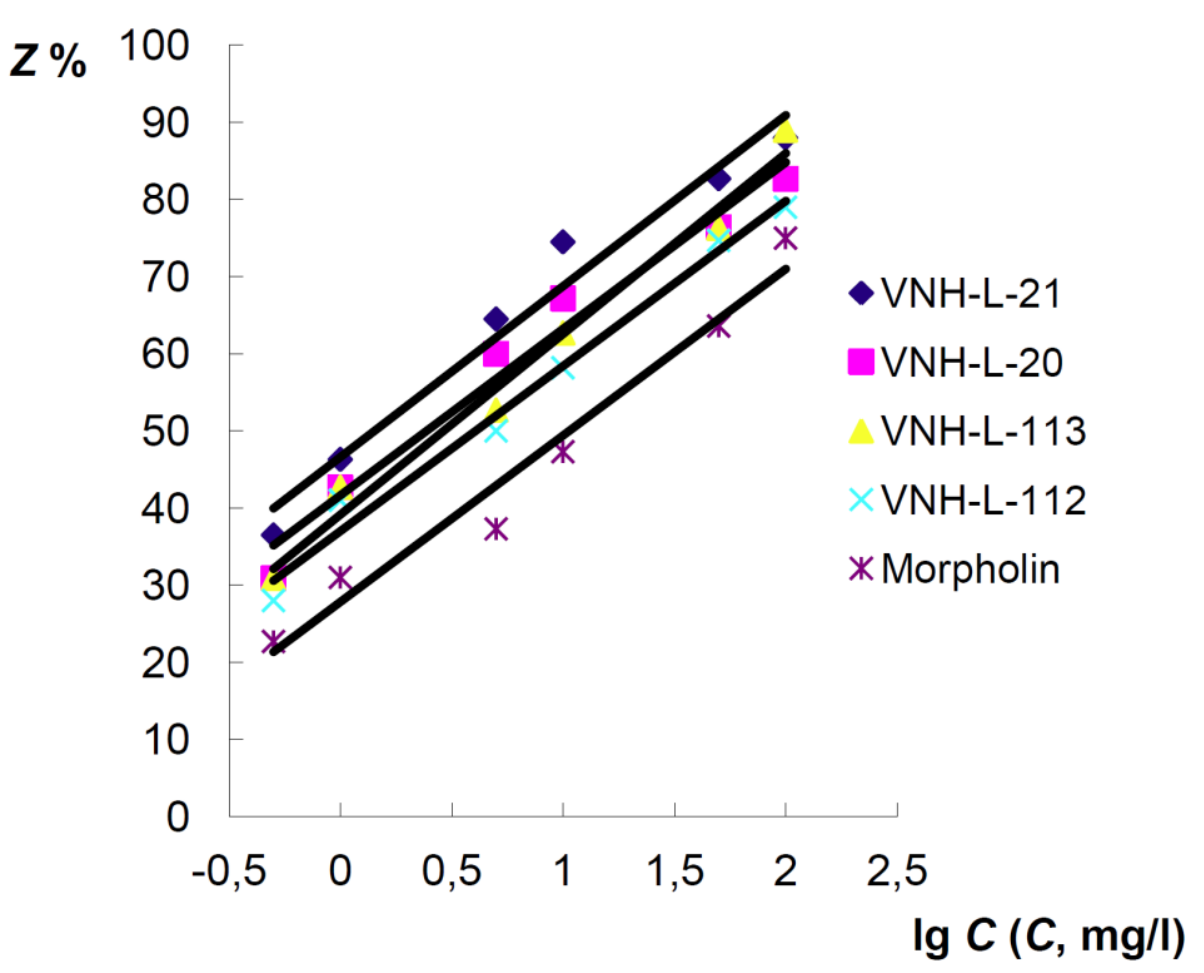

Figure 2. The dependence of the protective effect of morpholine and its derivatives on the concentration.

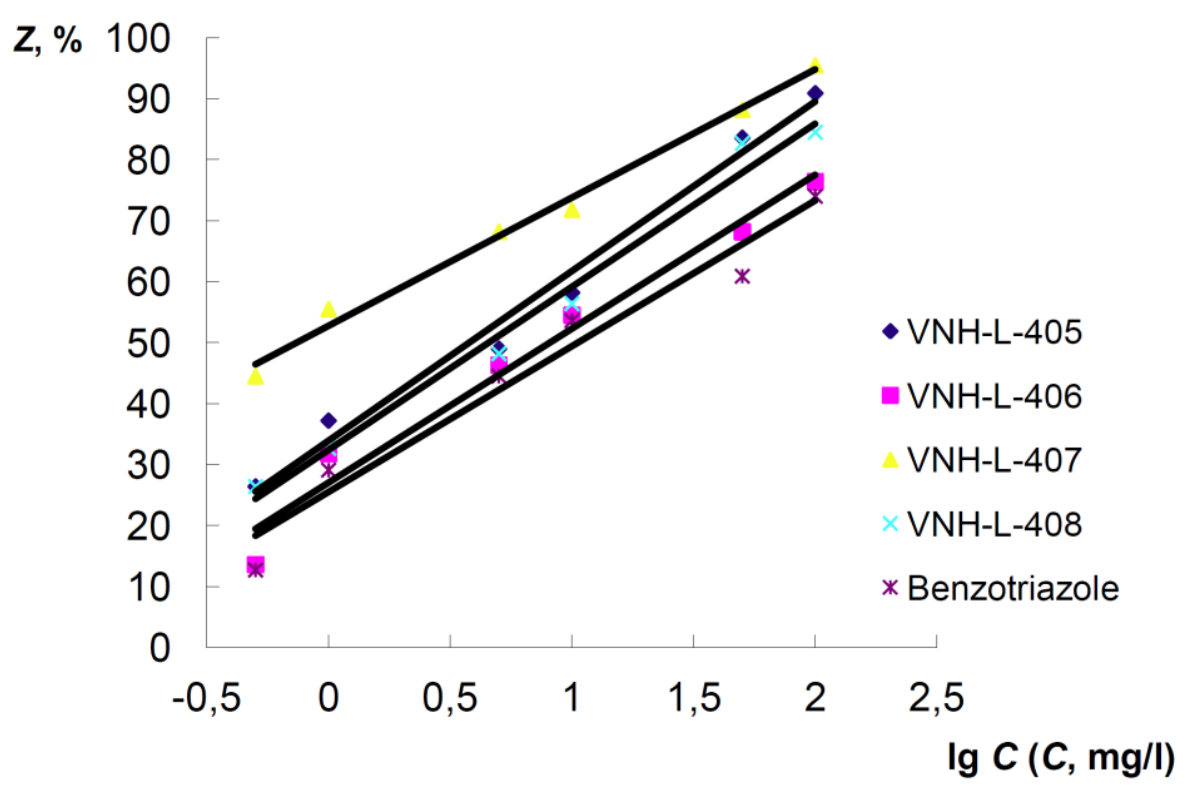

Figure 3. The dependence of the protective effect of benzotriazole and its derivatives on the concentration.

It was previously shown that the limiting stage of anodic dissolution is the incorporation of metal ions into the structure of the near-electrode layer of water molecules [5]. When organic inhibitors are added to the electrolyte, they are adsorbed at the metal - 
electrolyte interface [6], which leads to a decrease in the double layer capacitance due to a decrease in the dielectric constant. As a result, iron ions formed during anodic dissolution, are introduced into the medium with a dielectric constant lower than water, which leads to an increase in the activation energy of the electrochemical reaction of anodic dissolution [7]:

$$
E_{\mathrm{a}}=N_{\mathrm{a}}(\Delta e)^{2}\left(\frac{1}{\varepsilon_{0}}-\frac{1}{\varepsilon_{\mathrm{s}}}\right)\left(\frac{1}{2 a}-\frac{1}{4 R^{\prime}}\right)
$$

The result is an effective inhibition of the anodic dissolution reaction observed in the experiment.

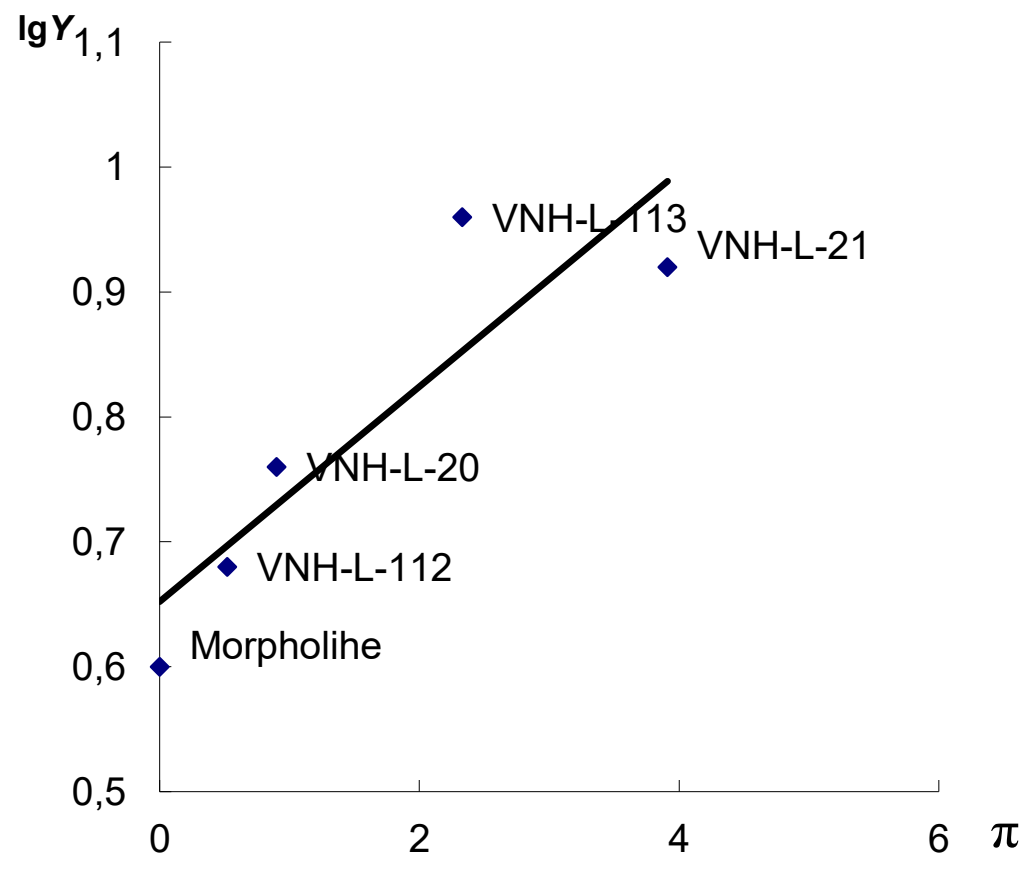

Figure 4. The dependence of the inhibition by morpholine and its derivatives on the Hansch constants. 


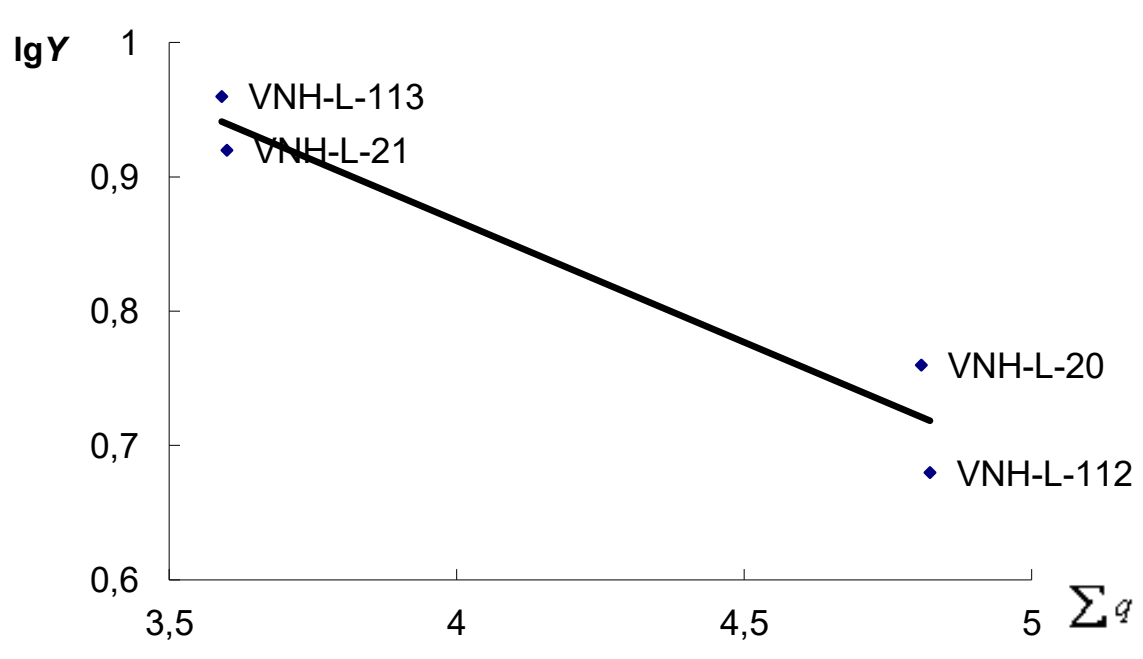

Figure 5. The dependence of the inhibition by morpholine and its derivatives on the electron density on the heteroatoms.

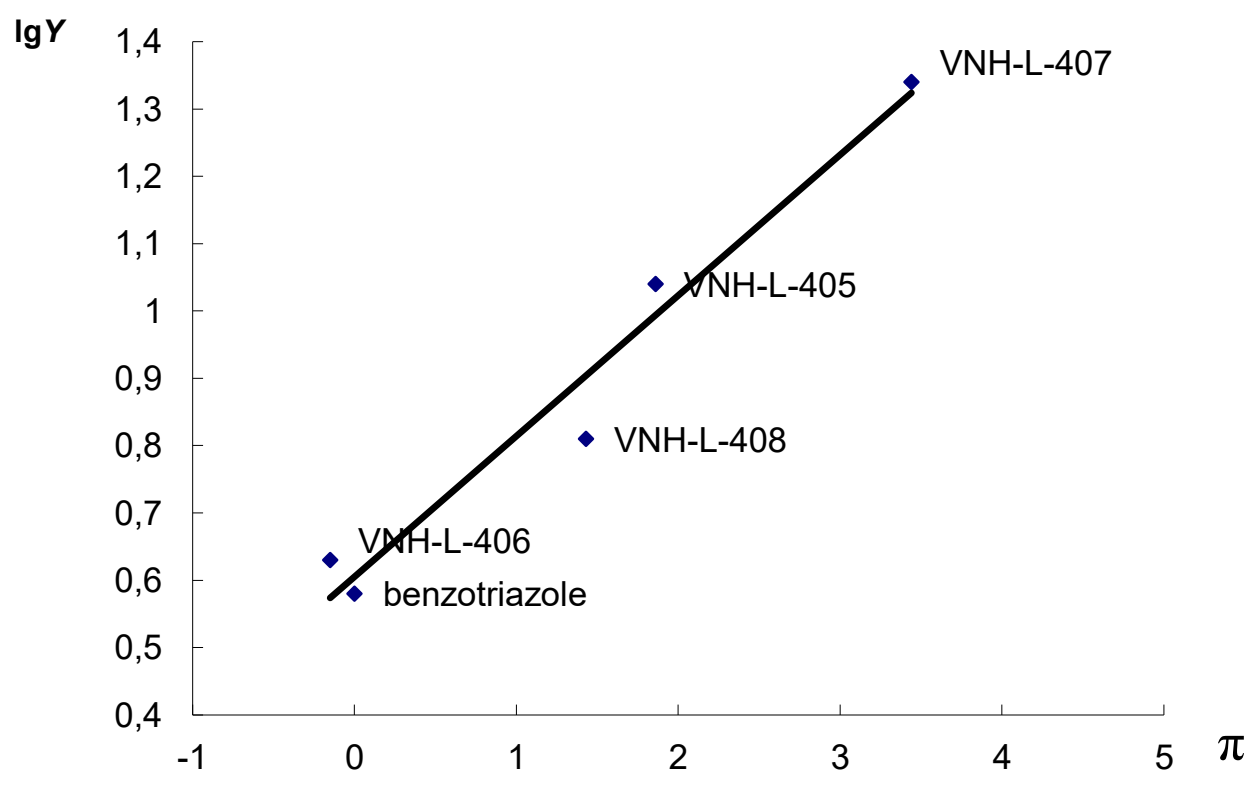

Figure 6. The dependence of the inhibition by benzotriazole and its derivatives on the Hansch constants.

\section{Conclusion}

Thus, the protective effect of the morpholine and benzotriazole derivatives studied in the present work during iron corrosion in a neutral medium is due to the effect of the hydrophobicity of inhibitor molecules. 


\section{References}

1. A.I. Altsybeeva, V.V. Burlov, N.S. Fedorova, T.M. Kuzinova and G.F. Palatik, Int. J. Corros. Scale Inhib., 2012, 1, no. 1, 51-64. doi: 10.17675/2305-6894-2012-1-1-051-064

2. N.N. Andreev, Yu.I. Kuznetsov and T.V. Fedotova, Zashch. Met., 2001, 37, 5 (in Russian).

3. A.I. Altsybeeva, V.V. Burlov, N.S. Fedorova and T.M. Kuzinova, Int. J. Corros. Scale Inhib., 2014, 3, no. 3, 160-166. doi: 10.17675/2305-6894-2014-3-3-160-166

4. Yu.I. Kuznetsov and L.P. Kazansky, Russ. Chem. Rev., 2008, 77, 219.

5. M.A. Pletnev and S.M. Reshetnikov, Zashch. Met., 2004, 40, 513 (in Russian). doi: 10.1023/B:PROM.0000043064.20548.e0

6. A.I. Altsybeeva, V.V. Burlov, N.S. Fedorova and S.M. Reshetnikov, Int. J. Corros. Scale Inhib., 2013, 2, no. 4, 277-286. doi: 10.17675/2305-6894-2013-2-4-277-286

7. I.O. Shapiro, Fizicheskaya Khimiya. Sovremennye problemy (Physical chemistry. Contemporary problems), 1987, Ed. Acad. Ya.M. Kolotyrkin, Khimiya, Moscow, 128 (in Russian). 